hep-th/9801134

CERN-TH/97-377

IASSNS-HEP-98/6

\title{
On the Realization of Chiral Four-dimensional Gauge Theories Using Branes
}

\author{
Amihay Hanany \\ School of Natural Sciences \\ Institute for Advanced Studies \\ Princeton, NJ 08540, USA \\ hanany@ias.edu \\ Alberto Zaffaroni \\ Theory Division, CERN \\ CH-1211 Geneve 23, Switzerland \\ Alberto.Zaffaroni@cern.ch
}

We consider a general brane construction for realizing chiral four-dimensional gauge theories. The advantage of the construction is the simplicity and the possibility of realizing a large class of models existing in the literature. We start the study of these models by determining the matter content and the superpotential which naturally arise in the brane construction.

CERN-TH/97-377

December 97 


\section{Introduction}

Configurations of branes have provided a useful tool for analysing non-perturbative properties of supersymmetric gauge theories in various dimensions. The problem of constructing general $N=1$ chiral gauge theories in four dimensions and of studying related problems, such as dynamical supersymmetry breaking, is still open.

In the spirit of the construction that was initiated in [1], there are currently three different ways to construct chiral gauge theories. In [2], chiral symmetry was found at special points in the brane realization of $N=1$ supersymmetric QCD. This led to a localization of chiral matter in space, which was done in [3], but produced only nonchiral theories. In this approach, more general constructions lead to chiral theories [4, [4, 6]. Another construction was made in [7], using the four-dimensional version of the theory in [1] sitting at an orbifold singularity. In this way, the theory $S U(N)^{k}$ with gauge factors and chiral matter associated respectively with the nodes and the links of the extended Dynkin diagram for $A_{k-1}$ was realized. The theory is chiral in the sense that each one of the bi-fundamentals connecting neighbouring factors is a chiral representation of both the gauge groups under which it is charged. However, each $S U(N)$ factor contains the same number of fundamental and anti-fundamental representations. More general theories containing tensor representations for the various groups were obtained in [8] by introducing orientifold planes in the picture. The theories that can be obtained in this way are the $N=1$ relatives of the theories classified in [9] (see also [10,11, 12] for generalizations).

In this paper we would like to report on a third approach to construct chiral gauge theories in this spirit. Following an approach used in [13] (see also [14]) to study sixdimensional theories, we will consider a T-dual version of these theories, which allows more flexibility in building models. The construction of particular models with tensor representations exists in the literature (see, for example, [8, 国, 5, 6]. ), but since a unified picture in which we can realize a larger class of models is still missing, we show in this note how to realize a big number of chiral models, leaving for future work the more detailed analysis of their dynamical properties. We will discuss what kind of superpotential is naturally present in the brane picture. We will obtain the matter content and the superpotential of several models, which are supposed to break supersymmetryl.

1 For a recent paper dealing with the issue of supersymmetry breaking in non-chiral models constructed with branes, see [15]. 
The three approaches to the construction of chiral gauge theories are presumably related by a sequence of T- and S-dualities. Below, we describe a T-duality between the second and the third. It will be interesting to check the precise relation and to learn more on these constructions by following the duality transformations.

We will use a mechanism proposed in [7], which is quite general. Start with a D-brane realization of an $N=2$ (minimal) supersymmetric gauge theory in five dimensions. We can obtain such a five-dimensional theory in several ways, using, for example, D4-branes at orbifold singularities [9] in Type IIA, or webs of $(p, q)$ five-branes in Type IIB [16]. The introduction of NS-branes, which limit the world-volume of the D-branes in the fifth direction, following the proposal in [1], induces a KK reduction to four dimensions, projects out the fields that correspond to motion in the directions transverse to the NS-brane and generally further breaks $N=2$ to $N=1$. For the Type IIA picture (D4-branes at orbifold singularities), the hypermultiplet matter content of the $N=2$ theory usually parametrizes the fluctuations of the D-branes in four spacetime directions. If the world-volume of the NS-branes is carefully chosen so as to freeze two of these four directions, the $N=2$ hypermultiplet is projected out to an $N=1$ chiral multiplet. We will consider the Type IIB realization in this paper.

There are several papers [8,4, 5, 6] that deal with the brane construction for chiral theories. Some of the models in these papers can be connected to our construction by an explicit T-duality. One of the advantages of the realization presented in this paper is its simplicity and the possibility of obtaining a very large class of models using a unified construction. Classical flat directions are easily studied in this approach, as in [1], and may be helpful in cases where the field theory analysis gets complicated.

\section{Building blocks for chiral theories}

A convenient way to realize an $N=1$ chiral gauge theory is to start with a fivedimensional minimally supersymmetric $N=2$ theory, constructed with a web of five-branes in Type IIB [16], and to project down to $N=1$ in four dimensions by introducing two extra five-branes that act as boundaries in the fifth direction 2. The previous statement is quite unrigorous. If we try to apply the set-up of [1] in five dimensions, we immediately realize that an NS-brane, having the same number of dimensions as a D5-brane, cannot behave

2 Such configurations were first considered in 17. 
as a rigid boundary that would absorb the RR charge of the D5-branes. When a D5 and an NS-brane touch [16], they merge in a $(1,1)$ brane, which extends in a direction dictated by supersymmetry. Therefore, the general system is realized with $(p, q)$ five-branes that intersect in such a way as to preserve charge and with angles dictated by supersymmetry [16]. We can determine the branes that, in the spirit of [1], act as boundaries by looking at the behaviour of the system at spatial infinity. The introduction of two extra five-branes to break supersymmetry down to $N=1$ further complicates the construction. To simplify the description of the model and the determination of the gauge and matter content, we will work in this section with zero string coupling, $g_{s}=0$ and zero axion $\chi=0$. This implies that we can consider the NS-branes as infinitely rigid boundaries, which absorb the charge of the D5-branes without being bent. The difference in tension of the two types of branes can justify this assumption. To see this in more detail we consider the asymptotic orientation of a $(p, q)$ five-brane, which is restricted by the condition of supersymmetry. Suppose that the D5-brane is stretched along a direction $x$ and the NS-brane is stretched along a direction $y$. The D5-brane is point-like in $y$ and the NS-brane in $x$. All other directions are shared by the two branes. Then, a $(p, q)$ five-brane preserves the same amount of supersymmetry, provided it is stretched as a line in the $(x, y)$ plane with a slope given by 18

$$
\Delta x: \Delta y=p+q \tau
$$

where $\tau=\frac{i}{g_{s}}+\frac{\chi}{2 \pi}$. In a background in which the Type IIB axion and the string coupling are zero, any $(p, q)$ five-brane with $q \neq 0$ will be parallel to the $y$ direction, whereas any $(p, 0)$ five-brane will be parallel to the $x$ direction. This gives a support for the assumption that the NS-branes can be considered rigid and do not bend when D5-branes end on them. This assumption simplifies greatly the discussion about the classical field theory with its matter content and classical interactions. At a later stage we would like to take the string coupling to be non-zero and then deduce information on the quantum dynamical properties of the gauge theory studied. But, even within this classical approximation, we will be able to make some statements on the IR properties of the gauge theories we constructed.

In conclusion, in this section we consider the naive T-dual of the model in [1] in the presence of two extra NS-branes (which we will call NS'). The ingredients are: D5branes with world-volume (012346), NS-branes with world-volume (012345), NS' branes with world-volume (012367), and D7-branes and O7 orientifold planes with world-volume (01234789). The two types of NS-branes bound the D5-branes in both directions 4 and 
6 , and the KK reduction in these two directions gives a four-dimensional theory. The D5 world-volume in 46 appears, in the approximation we are considering, as a rectangle bounded by the NS-branes. In addition to the above branes, we may introduce other branes or singularities, which will not break the supersymmetry further. These are ALE space along (4567), D7' with world-volume (01235689) and D5' with world-volume (012357) [17]. We will not make much use of these additional branes in this paper.

The presence of these branes breaks space-time Lorentz symmetry from $S O(1,9)$ to $S O(1,3) \times S O(2)$. The first group is identified with the Lorentz symmetry of the fourdimensional theory studied, while the $S O(2)$ symmetry acts on the 89 directions and is identified with the $U(1)_{R}$ symmetry of the $N=1$ supersymmetric system.

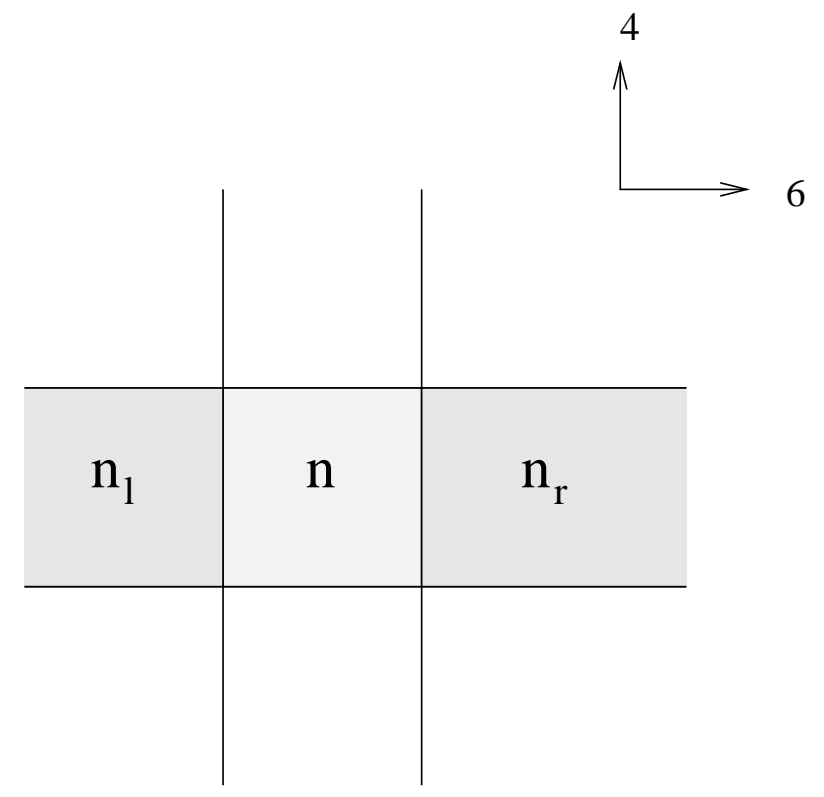

Figure 1: Brane realization of $S U(n)$ gauge theory with $n_{l}$ chiral multiplets in the fundamental representation and $n_{r}$ chiral multiplets in the antifundamental representation. The horizontal lines represent $\mathrm{NS}^{\prime}$ branes and the vertical lines represent NS-branes. between them there are $n$ D5-branes bounded by the box. There are also semi-infinite D5-branes to the left and to the right.

The basic building block is obtained in the following way. Start, as in fig. 1, with a theory with 8 supercharges obtained by stretching $n$ D5-branes along the direction $x_{6}$ between two NS-branes and putting also $n_{l}$ semi-infinite D5-branes on the left and $n_{r}$ on the right. This is the five-dimensional minimally supersymmetric theory of an $S U(n)$ gauge group with $n_{l}+n_{r}$ hypermultiplets. Introduce further two NS'-branes, which bound the D5-branes at a finite distance in $x_{4}$. The hypermultiplets parametrize fluctuations 
of the D5-branes in 6789, but the presence of the $\mathrm{NS}^{\prime}$ freezes the possible motions in 67. The $N=2$ hypermultiplets are projected down to $N=1$ chiral multiplets, with different chirality depending on their position to the left or to the right. The field content is therefore $S U(n)$ with $n_{l}$ fundamentals and $n_{r}$ antifundamentals. Anomaly cancellation requires that $n_{l}=n_{r}=n_{f}$. It would be interesting to see how this arises from $\mathrm{RR}$ charge conservation.

The parameters of the theory are given by the positions of the two types of NSbranes. There are 4 NS-branes with 4 transverse positions to each, which gives 16 possible parameters. Six parameters can be set to zero by tuning the origin in directions 456789 . We are left with 10 parameters. There are 4 distances in the 4567 directions and there are 3 positions in the 89 directions. The gauge coupling is given by the area of the rectangle in the 46 directions:

$$
\frac{1}{g^{2}}=\frac{\Delta x_{4} \Delta x_{6}}{g_{s} l_{s}^{2}} .
$$

The other parameters do not have a clear interpretation. The distances in the 5 and 7 directions look like FI terms for the $U(1)$ gauge groups. Since it is frozen from a fourdimensional point of view, they may be promoted to moduli of the field theory. The same comment applies for the other positions in the directions 89. An analysis of specific cases makes their interpretation clearer, mostly as dynamical moduli.

The theta angle is related to the Type IIB axion. The D5-brane has a term in the effective action, which looks like

$$
\frac{\chi}{2 \pi} F \wedge F \wedge F
$$

Integrating over the rectangle in the 46 directions, the four-dimensional theta angle is given by

$$
\frac{\theta}{2 \pi}=\frac{\chi}{2 \pi} \int_{46} F
$$

Let us study some classical flat directions. Let us denote the fundamental fields by $Q_{i}$ and the antifundamental fields by $\widetilde{Q}^{j}$. We can reconnect a left semi-infinite D5-brane to a finite D5-brane and to a right semi-infinite D5-brane. They form an infinite D5-brane in the 6 direction. The D5-brane is now free to move between the two $\mathrm{NS}^{\prime}$ branes in the 7 direction. What remains are $n-1$ finite branes with $n_{f}-1$ semi-infinite branes to the left and to the right. The gauge group is broken to $S U(n-1)$ with $n_{f}-1$ flavours. Such a motion corresponds to a non-zero expectation value for a meson field, say $\widetilde{Q}^{1} Q_{1}$. One can generalize this to $r$ such branes. The gauge group is broken to $S U(n-r)$ with $n_{f}-r$ 
flavours. The positions of the branes parametrize the eigenvalues of the mesonic matrix $M_{i}^{j}=\widetilde{Q}^{j} Q_{i}$. For this case, $r$ of them are non-zero. For $n_{f} \geq n$, there is also a baryonic branch. This corresponds to reconnecting at one side, say the right, $n$ of the semi-infinite D5-branes. The $n_{f}-n$ remaining semi-infinite branes can now move, together with the right NS-brane, along the 7 direction. The distance in the 7 direction corresponds to the expectation value of the baryons. This case serves as an example to the comment made in the last paragraph on distances between NS-branes.

One may question that the matter localized at the intersection of two D5-brane along the NS-branes bounded by NS'-branes is indeed chiral and not hyper. The agreement of the classical moduli space with the field theory in question serves as a support for this identification.
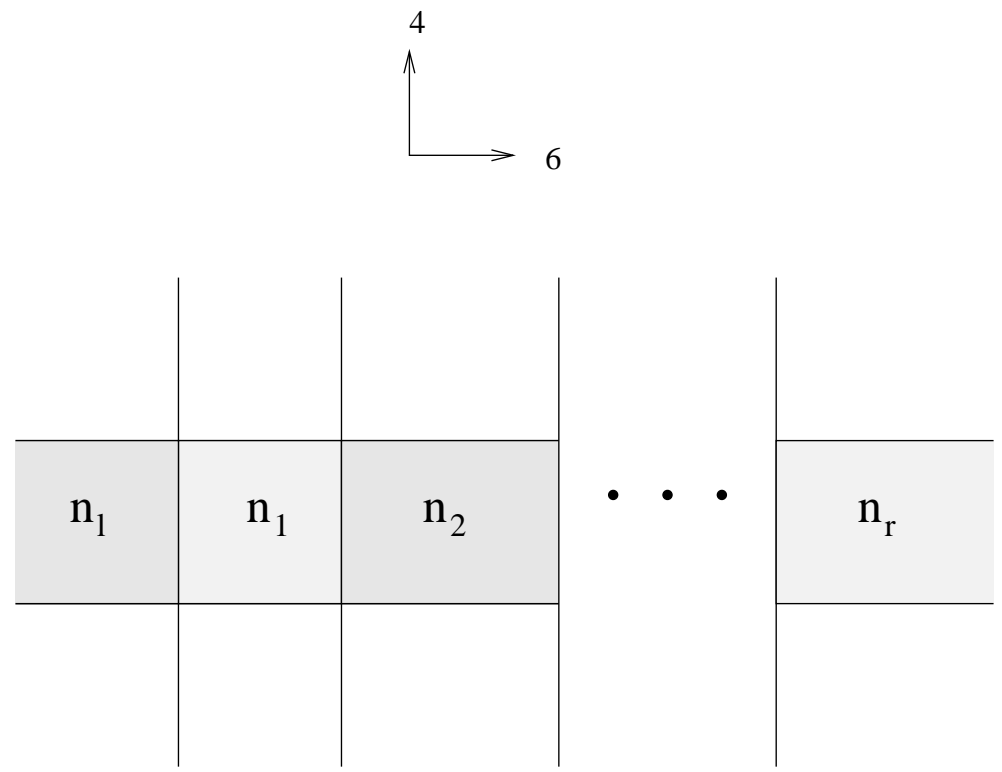

Figure 2: Brane realization of product of $S U$ gauge groups. The horizontal lines represent $\mathrm{NS}^{\prime}$ branes and the vertical lines represent NS-branes. There are $n_{l}\left(n_{r}\right)$ semi-infinite D5-branes to the left (right). Finite D5-branes are bounded by the NS-branes and the NS'-branes. The number of D5-branes is denoted in each box. The different shaded regions are to emphasize that the number of D5-branes can be different.

It is easy to generalize the theory to a product of $S U$ gauge factors. Consider, as in fig. 2, $P$ NS-branes displaced along the direction $x_{6}$, with $n_{i}$ D5-branes in between the $i$-th and $(i+1)$-th NS-brane and $n_{l}$ semi-infinite D5-branes on the left and $n_{r}$ on the right. The $N=1$ gauge theory is

$$
\prod_{i=1}^{P-1} S U\left(n_{i}\right)
$$


with chiral bifundamentals charged under each neighbouring factor, and extra $n_{l}$ fundamentals for $S U\left(n_{1}\right)$ and $n_{r}$ antifundamentals for $S U\left(n_{P-1}\right)$.

For general values of $n_{i}$ the theory is obviously anomalous. Charge conservation for the RR spacetime fields should be correspondingly violated. Since we have discarded the bending of the five-branes and the issue of the charge conservation at the intersection of various branes, we cannot see this spacetime phenomenon explicitly. For the moment, we use the field theory input. There are two series of anomaly-free models. The first one has $n_{l}=n_{i}=n_{r}=n$,

$$
\prod_{i=1}^{P-1} S U(n)
$$

with chiral bifundamentals, $n$ fundamentals for the first factor and $n$ antifundamentals for the last. This is the T-dual of the model in [7], which can be exactly reproduced if we take a compact $x_{6}$ direction. The second anomaly-free model has the following field content:

$$
S U(n) \times S U(m) \times S U(n) \times S U(m) \times \ldots
$$

with chiral bifundamentals and $m$ fundamentals for the first factor. The last factor can be $S U(n)$ with $m$ antifundamentals or $S U(m)$ with $n$ antifundamentals.

We can introduce extra matter using D7-branes. Each of them produces a hypermultiplet in the $N=2$ theory coming from the open strings between D5- and D7-branes. Since the intersection between D5- and D7-branes is, in general, localized far from the D5 boundaries, the full multiplet, which decomposes in two chiral multiplets of the $N=1$ theory, survives the projection imposed by the NS-branes. We will give further consistency checks on this identification in the next section by using brane motion and creation. The D7-brane gives rise to two scalars, positions in the 56 directions. The position in the 5th direction gives rise to a mass for the quark multiplet. Together with the Wilson line along the direction 4 of the D7-brane, they combine into a chiral multiplet, which gives rise to the mass of the quark fields.

The superpotential of the model is derived from the local $N=2$ supersymmetry. It gives a Yukawa coupling with an adjoint field and two quark fields. However, the adjoint scalar involved is frozen by the bounding by NS-branes. This leads to a configuration with no superpotential. A mass term for the quarks still exists. The superpotential can be modified by introducing a rotation of the D7-branes in the directions 47-56. This changes the coefficient of the Yukawa coupling as in cases discussed in [16]. 
We can construct higher-order representations by introducing an orientifold plane parallel to the D7 in the picture. There are two possible choices of sign for the charge of such an orientifold plane: in the case of a negative sign, which arises in the standard Type $\mathrm{I}^{\prime}$ string theory, we call the orientifold $\mathrm{O}$, while in the case of a positive sign, we call it $\mathrm{O}^{+}$. Every D- or NS-brane now must have an image under the $Z_{2}$ symmetry $x_{5,6} \rightarrow-x_{5,6}$ or be stuck at the orientifold point. We will take the two NS' stuck at $x_{5}=0$ and the NSbranes disposed in $x_{6}$ in such a way as to preserve the $Z_{2}$ symmetry. There are essentially two basic configurations [19,13,,14],
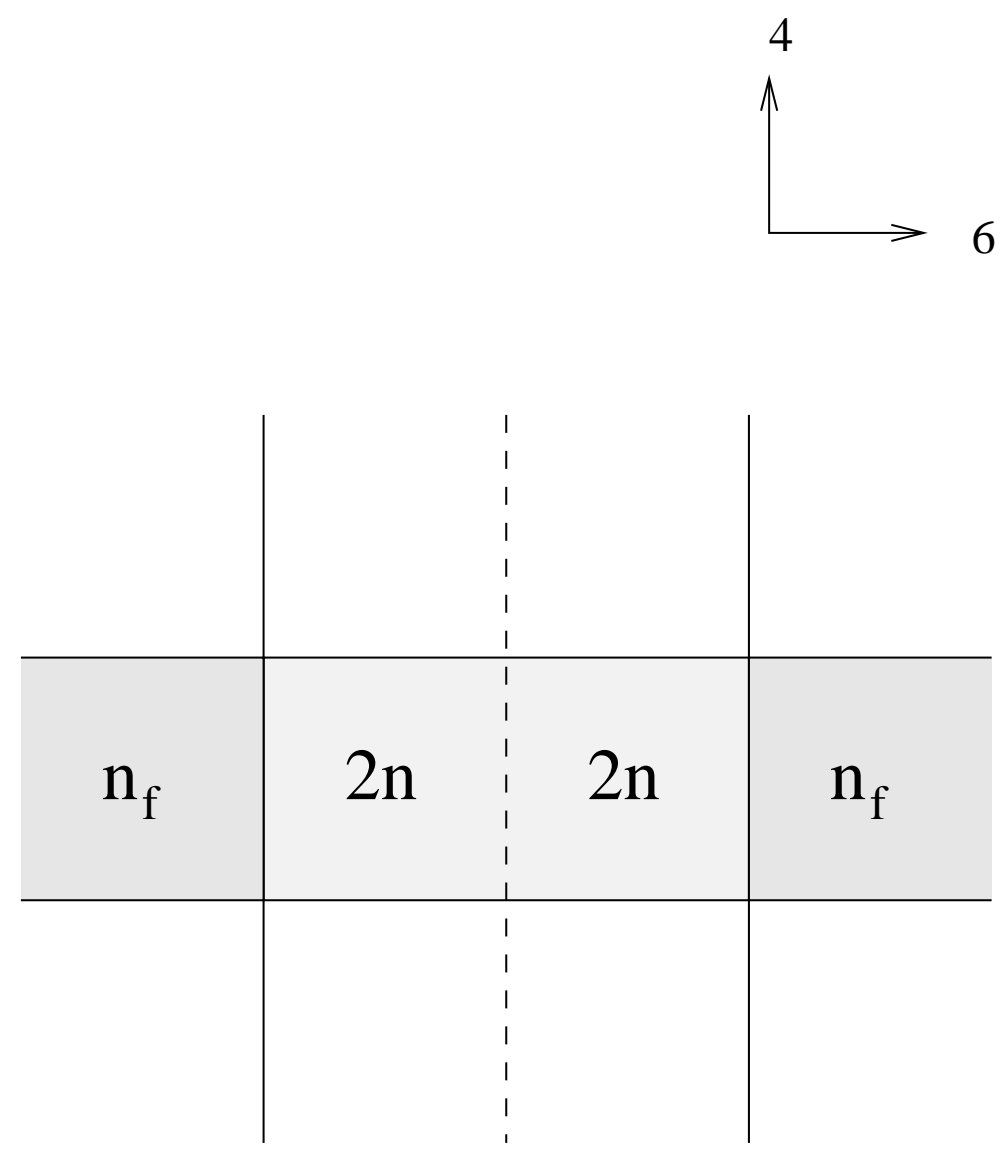

Figure 3: $S p(n)(S O(2 n))$ gauge group with $n_{f}$ flavours. The dashed line represents an $\mathrm{O} 7\left(\mathrm{O}^{+}\right)$plane. $n_{f}$ semi-infinite D5-branes give rise to $n_{f}$ chiral multiplets.

1) Consider, as in fig. 3, an NS-brane at $x_{6}^{0}$ and stretch $2 n$ D5-branes between it and its image at $-x_{6}^{0}$. Put also $n_{f}$ semi-infinite D5-branes on the right of the NS-brane. The two NS'-branes project down the theory to $\operatorname{USp}(2 n)$ (for $\mathrm{O} 7)$ or $S O(2 n)\left(\right.$ for $\mathrm{O}^{+}$) with $n_{f}$ fundamentals. In the $\mathrm{O}^{+}$case, there is the option to have an odd number of D5-branes, one of them being stuck, giving the group $S O(2 n+1)$. 
2) Consider an NS-brane stuck at $x_{6}=0$ and stretch $n$ D5-branes in between it and a second NS-brane at $x_{6}^{0}$. Put also $n_{f}$ semi-infinite D5-branes on the right of the second NS-brane. Since the D5-branes are identified with their images (D5-branes stretched between the stuck NS-brane and the image of the second NS at $\left.-x_{6}^{0}\right)$, no projection on the Chan-Paton factors is needed and the gauge group is $S U(n)$. The open strings connecting the D5-branes with their images give rise to a multiplet in the antisymmetric representation of the gauge group (for O7) or in the symmetric one (for $\mathrm{O}^{+}$)[19]. In conclusion, the theory is $S U(n)$ with an antisymmetric (symmetric) and $n_{f}$ antifundamentals.

The theory in 1) is well defined for all values of $n$. Global anomalies of $S p$ gauge theories give rise to a constraint on $n_{f}$. In the case of $O 7, n_{f}$ must be even. For the case of $\mathrm{O}^{+}$, the gauge group is $S O$ and there is no restriction on $n_{f}$. For the theory 2 ), anomaly cancellation requires $n=n_{f}+4$ (for O7) and $n=n_{f}-4\left(\right.$ for $\left.7^{+}\right)$.

We can now summarize two rules for D5-branes stretched between a pair of NS-branes and a pair of $\mathrm{NS}^{\prime}$-branes. The rules are derived from the field theory requirement that the anomalies will be cancelled. It would be nice to get this rule from an independent argument, which does not rely on the field theory analysis. Nevertheless, we shall state the rules and study the consequences of these two rules below. We hope to return to an independent derivation of these rules in the future. The rules are:

a) Given, as in fig. 1, $n$ D5-branes between two NS-branes and two NS'-branes with $n_{l}\left(n_{r}\right)$ D5-branes to the left (right), the consistency condition requires that $n_{l}=n_{r}$.

b) Given, as in fig. 3, $n$ D5-branes between NS-brane and its image under an $\mathrm{O} 7\left(\mathrm{O}^{+}\right)$ and between two $\mathrm{NS}^{\prime}$-branes with $n_{f}$ D5-branes connecting along the NS-branes, the consistency condition requires that $n_{f}=n-4(n+4)$. We will use these rules below.

Generalizations can be obtained by adding other NS-branes (and their images under $Z_{2}$ ). If $n$ D5-branes are stretched between two NS-branes that are not stuck at the orientifold, the $Z_{2}$ projection identifies them with their images living on the other side of the orientifold, without further projecting the Chan-Paton factors. Therefore they give rise to $S U(n)$ gauge groups. The generalization of the theories in 1) and 2) (for O7) reads, respectively,

$$
\begin{aligned}
& U S p\left(V_{0}\right) \times S U\left(V_{1}\right) \times S U\left(V_{2}\right) \times \ldots \\
& S U\left(V_{0}\right) \times S U\left(V_{1}\right) \times \ldots
\end{aligned}
$$


with chiral bifundamentals for each neighbouring gauge group, an antisymmetric for the factor $S U\left(V_{0}\right)$ and $n_{f}$ antifundamentals for the last factor if we put $n_{f}$ semi-infinite D5branes. In all the above cases, the groups $U S p$ become $S O$ if we use $\mathrm{O}^{+}$.

We conclude this section by discussing the relation of the Type IIB construction with the one considered in [7, [] using branes at orbifold singularities. We will see that an explicit $\mathrm{T}$ duality maps the models in [7,:8] in a subset of the theories we have considered in this section.

The theory in [7] is obtained by starting with $N$ D4-branes at a $Z_{k}$ orbifold singularity. We take the world-volume of the D4-branes to extend in the directions (01234) and the orbifold projection to act on (6789). The world-volume theory was determined in [9] and is associated with the extended Dynkin diagram for $A_{k-1}$ : each of the nodes provides an $S U(N)$ gauge factor and each of the links a hypermultiplet in the bifundamental of the two gauge groups corresponding to the nodes connected by the particular link. The hypermultiplets parametrize the fluctuations of the D4-brane positions in (6789). The introduction of two NS-branes with world-volume (012367) and different $x_{4}$ positions induces a KK reduction along the direction 4 , breaks the supersymmetry down to $N=1$ and freezes the 89 scalars in the hypermultiplets. Notice that the NS-branes must be stuck at $x_{8}=x_{9}=0$ to respect the $Z_{k}$ projection without introducing images and extra states in the theory. The surviving matter is composed by chiral multiplets parametrizing the motion in 67 . For each $S U(N)$ factor there are two such chiral multiplets corresponding to the two links that connect the given node to the two adjacent ones. They are fields in the fundamental representation of the gauge group, but with opposite chirality. The theory is anomaly-free since each gauge factor has the same number of fundamentals and antifundamentals.

Extra matter can be introduced using D8-branes with world-volume (012346789), which do not break any further supersymmetry. Each of them provides a pair of chiral multiplets in the fundamental and antifundamental representation. If we further introduce an $\mathrm{O} 8$ orientifold plane parallel to the D8-branes, the gauge and matter content is projected out according to the rules in [9]. The resulting theories have the form:

$$
\begin{aligned}
& U S p\left(V_{0}\right) \times S U\left(V_{1}\right) \times S U\left(V_{2}\right) \times \cdots S U\left(V_{P-1}\right) \times U S p\left(V_{P}\right), \quad k=2 P, \\
& U S p\left(V_{0}\right) \times S U\left(V_{1}\right) \times S U\left(V_{2}\right) \times \cdots S U\left(V_{P-1}\right) \times S U\left(V_{P}\right), \quad k=2 P+1, \\
& S U\left(V_{0}\right) \times S U\left(V_{1}\right) \times \cdots S U\left(V_{P-2}\right) \times S U\left(V_{P-1}\right), \quad k=2 P .
\end{aligned}
$$


The two cases for $k$ even correspond to different kinds of projections. The matter consists of chiral bifundamentals charged under each neighbouring gauge factor and of chiral antisymmetric for the first and last $S U$ factors 3 . It is convenient to perform a T-duality and transform the system to a brane configuration in Type IIB. This approach was already used in [13] and [14] to study six-dimensional theories.

If we perform a T-duality in $x_{6}$, the $Z_{k}$ orbifold singularity is converted into a set of $k$ NS-branes with world-volume (012345) positioned along $x_{6}$. The D4-branes becomes D5-branes that can break between the new NS-branes. The two old NS-branes with worldvolume (012367) remain unchanged. These are exactly the models we discussed before. It is easy to check that the gauge and matter content remains the same. The theories in (2.9) can be obtained by using a compact $x_{6}$ direction. The three different theories in (2.9) correspond to one odd case and two possible ways to put $2 P$ NS-branes on a circle in a $Z_{2}$ invariant way [13,14.

The Type IIB description is more general. Not all the realizations of models have known $\mathrm{T}$ duals in a description with branes at orbifold singularities. Nevertheless, the Type IIA picture is a complementary description and may be sometimes useful. In particular, the matter content and the superpotential of the Type IIA models can be explicitly computed using the orbifold projection. This provides non-trivial consistency checks about the Type IIB description. In the cases in which the theories have a known $\mathrm{T}$ dual we can check the correctness of the proposed matter content and superpotential by an explicit computation in the T-dual picture.

\section{Arrays of D5 boxes}

Another generalization is to consider a grid of $\mathrm{SU}$ theories as in fig. 4 . We take $P$ NS-branes and $R \mathrm{NS}^{\prime}$-branes. Let the matrix $N=\left\{n_{i j}\right\}, i=0, \ldots, P, j=0, \ldots, R$, denote the number of D5-branes in each box. Then, the gauge group is

$$
\prod_{i=1}^{P-1} \prod_{j=1}^{R-1} S U\left(n_{i j}\right)
$$

We expect chiral bifundamental matter at the intersection of any two boxes and chiral multiplets at the boundaries. Boxes can intersect along a line (for example, $n_{11}$ and $n_{12}$ ) or

3 The theories in [8] are obtained using an O4 plane instead of an O8 plane. The matter content of these theories is again classified in [9]. 

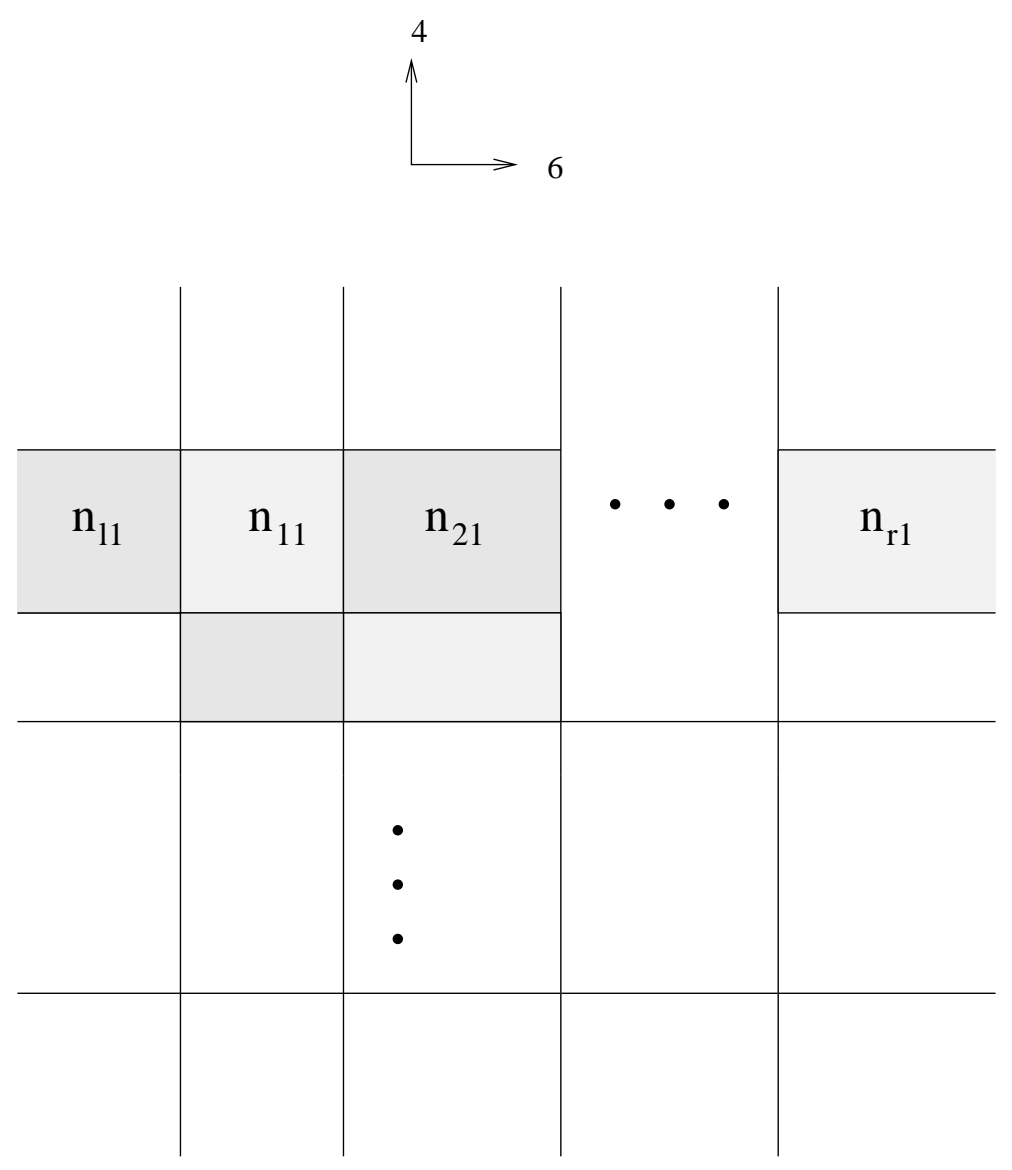

Figure 4: More general construction of a product of $S U$ gauge groups.

at a point (for example, $n_{12}$ and $n_{21}$ ). In both cases, we can expect chiral bifundamentals. The projection imposed by the NS-branes gives the following matter content for the model: there are, in horizontal, chiral bi-fundamentals $\left(n_{i, j}, \bar{n}_{i, j+1}\right)$, in vertical $\left(n_{i, j}, \bar{n}_{i+1, j}\right)$ and along the diagonal $\left(\bar{n}_{i j}, n_{i+1, j+1}\right)$.

Let us consider the three-box model of fig. 5. This model will serve as the basic building block for the models we will consider below. We can picture, as in fig. 5, the chiral bifundamentals that connect neighbouring boxes with arrows. Their direction specifies the chirality of the bifundamental. The rule is that the arrows can only be drawn in the direction East, North and South-West. The gauge group is $S U(k) \times S U(n) \times S U(m)$. The boxes may have finite or infinite area, which will correspond to a gauge symmetry or a global symmetry, respectively. This property, however, does not enter into the present discussion. As the arrows indicate, there are three chiral multiplets $Q_{1}, Q_{2}, Q_{3}$, transforming in $(k, \bar{n}, 1),(1, n, \bar{m}),(\bar{k}, 1, m)$, respectively. These fields allow for a superpotential of the form

$$
W=Q_{1} Q_{2} Q_{3} .
$$




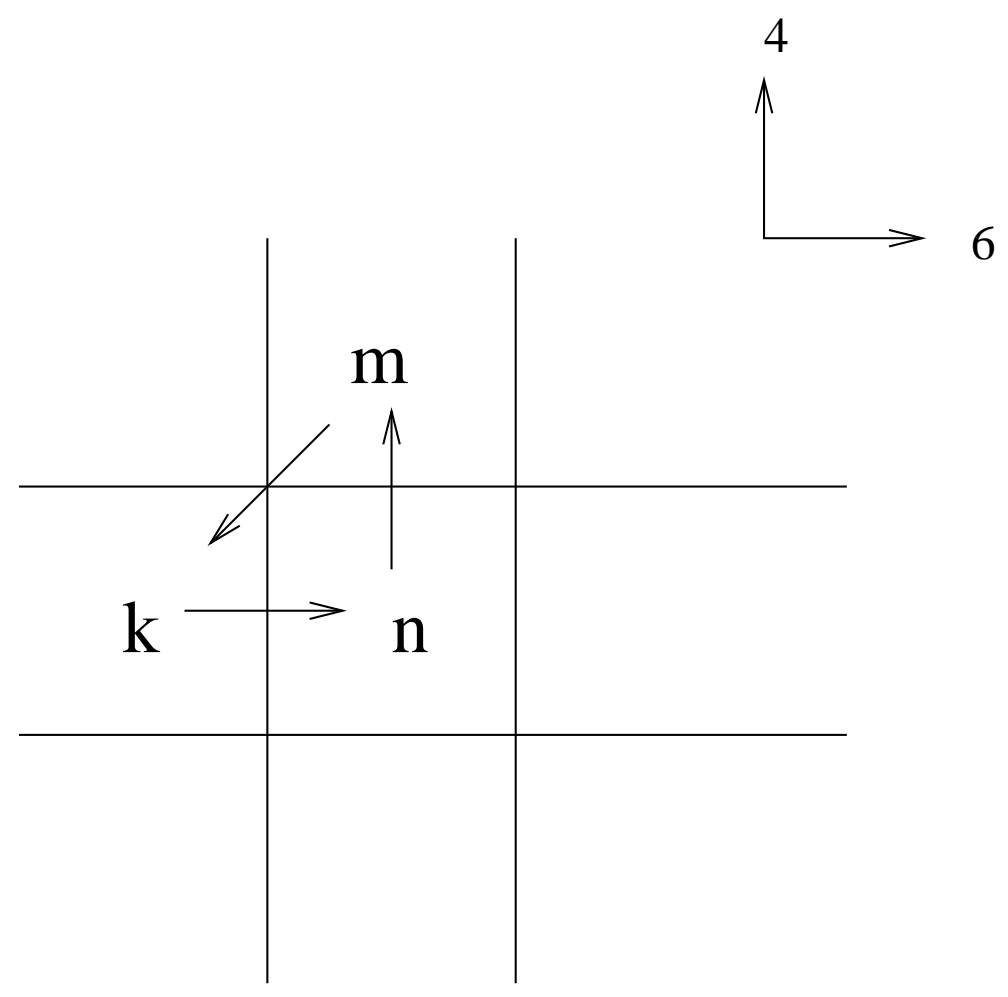

Figure 5: A three-box model with three groups, three chiral multiplets and a cubic superpotential. The number of D5-branes in each box is denoted by an integer. Empty boxes have no bound D5-branes.

Why is this the matter content? Previous experience with simple models such as those in fig. 2 teaches us about the horizontal bi-fundamentals and, using the symmetry of the construction, about the vertical ones. The presence of diagonal fields is more subtle. There are several arguments for their existence and to explain why only the fields associated with one of the two diagonals survive. First, recall that the chiral multiplets are given by strings that are stretched between the two D5-branes. In the absence of NS-branes (here, we shortly refer to either an NS or an $\mathrm{NS}^{\prime}$-brane), two possible orientations are allowed for the strings, which correspond to two opposite chiral fields. The presence of NS-branes induces a particular orientation for the strings. This gives rise to a single chiral field, with a given chirality. A string can be only parallel to the NS-brane and not antiparallel. This is why only an orientation, say going east (the choice of orientation is a matter of convention, and is specified by choosing the orientation on the NS-branes), is allowed and not, say, going west. Such strings are parallel to the NS-branes (and not to the NS'-branes). Similarly, only an arrow going, say, north and not going south can produce a multiplet. The string is then parallel to the $\mathrm{NS}^{\prime}$-branes. The diagonal intersection is restricted by the 


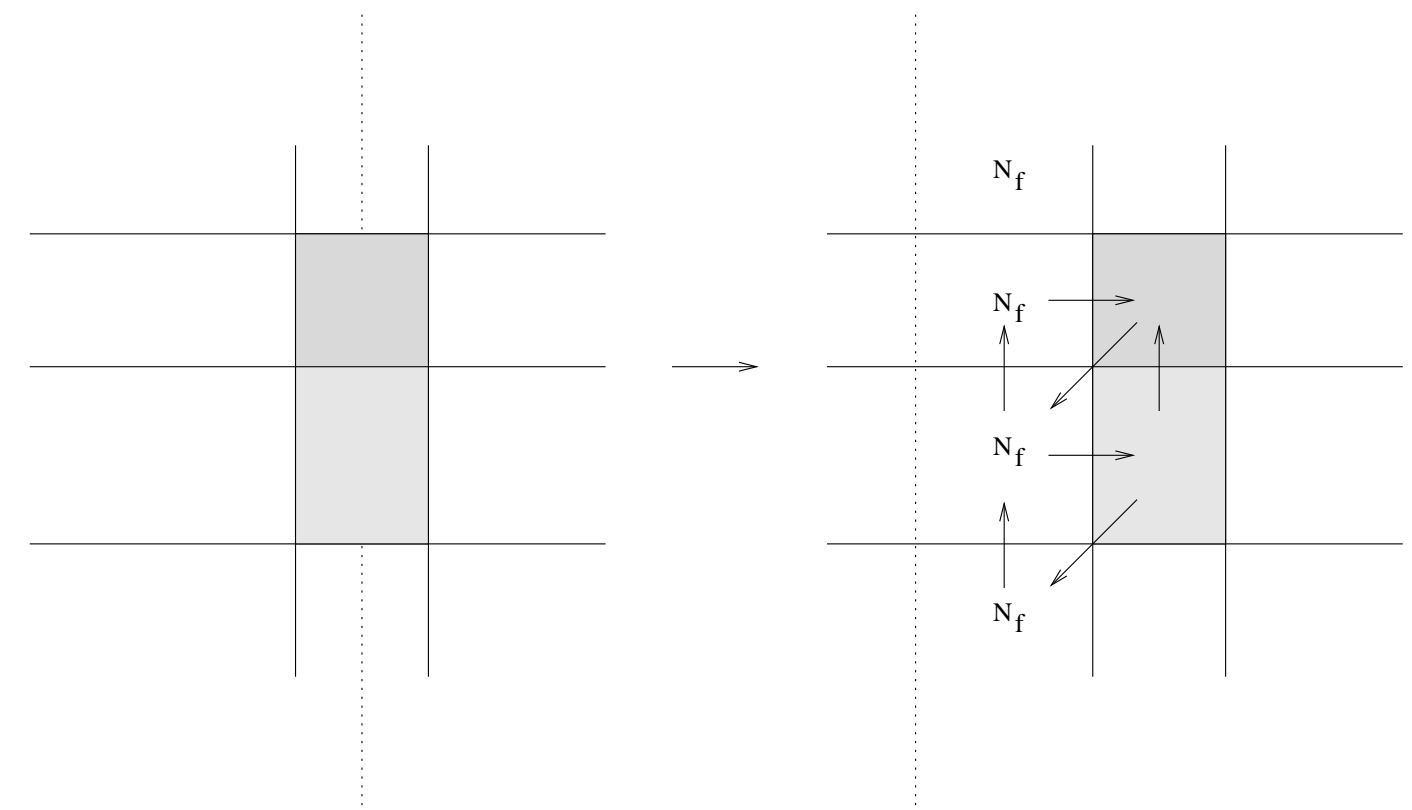

Figure 6: A creation of various boxes of D5-branes when a D7 and an NSbrane cross. The D7-brane is represented by a vertical dotted line.

orientation of both the NS- and NS'-brane and goes only south-west. In addition, since the three arrows form a closed circle, they give rise a superpotential, eq. (3.2).

A second argument goes as follows. Consider, as in fig. 6, the simple case in which we have a column of boxes, for example, $n_{i j}=\delta_{j} n$ for some $P$ with the addition of a D7-brane. The D7-brane provides vector-like matter for all the gauge groups. We can move the D7 to the left. When the D7 crosses the leftmost NS-branes, other D5-branes are created between it and the NS-brane [1]. Next move the D7 to infinity. The matter in the fundamental is now provided by semi-infinite D5-branes $n_{j-1, P}=1$. We shall assume, as in previous cases following [1], that the matter content does not change in this transition. There are chiral fundamentals provided by the semi-infinite D5-branes. To have a vectorlike matter as before the phase transition, we need antifundamentals coming from one and only one diagonal. The discussion on D7-branes is expanded in the next subsection.

As a third check, we notice that, by performing a T-duality, some of the models can be identified with the ones discussed in [7], where the matter content can be explicitly derived by an orientifold computation. The two results agree.

But perhaps the best motivation for the above matter content is the fact that the flat directions expected in field theory are exactly matched by the allowed motion of the branes. We will see several examples below. We must postpone the discussion until we specify the superpotential for all these theories. 


\subsection{Matter and Interactions from D\%-branes}

In this subsection we study in detail the introduction of D7-brane to the system. We find that there are, in addition to the expected results, new interactions that appear. For this reason, we devote this subsection to this effect.

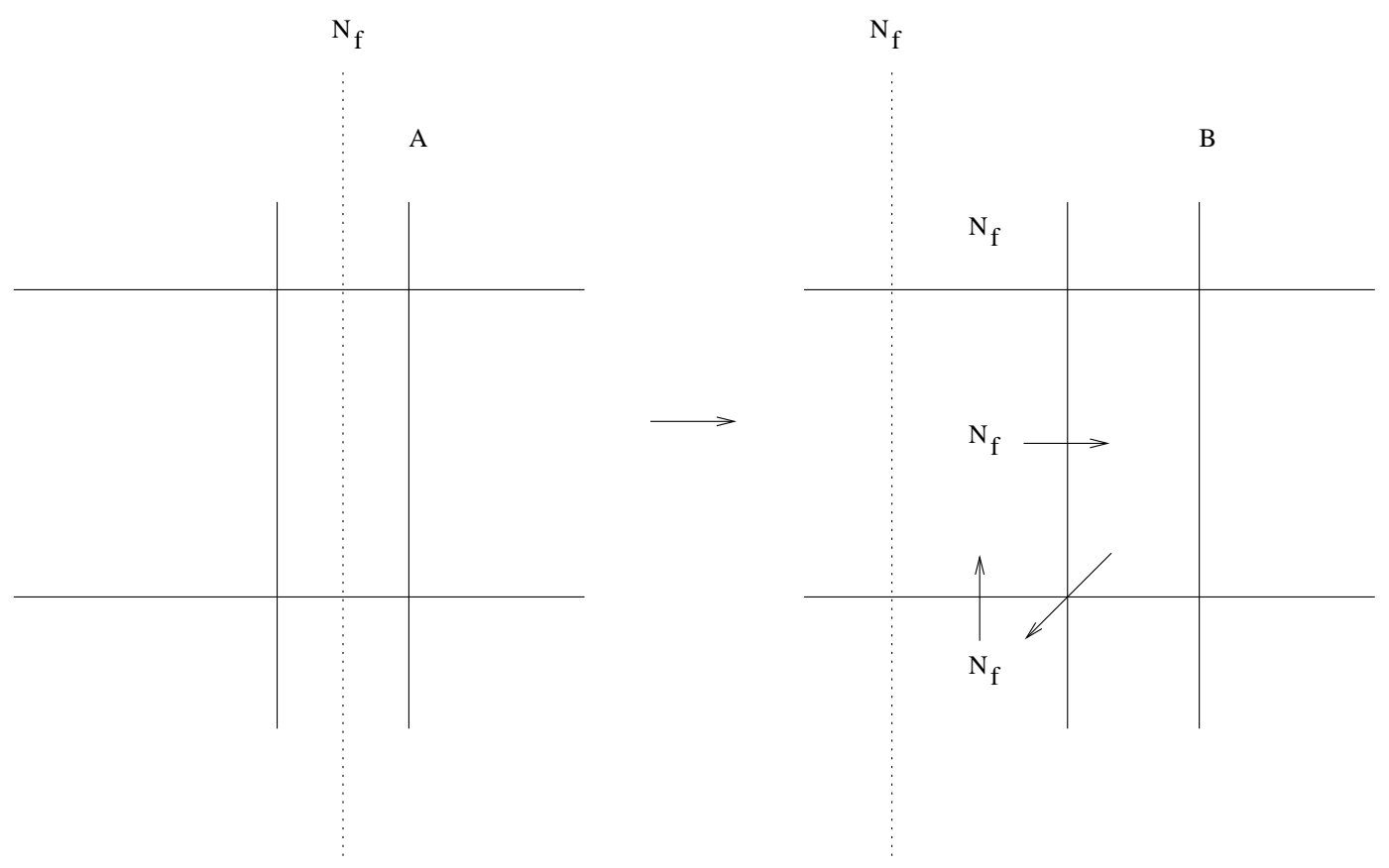

Figure 7: The creation of various boxes of D5-branes when a D7-brane and an NS-brane cross. The case with a single finite box. The arrows indicate all possible chiral fields relevant to the interaction between the D7-brane and the D5-branes.

Consider, as in fig. 7, a single box with $n$ D5-branes and a D7-brane. As in the usual case, the D7-brane gives rise to two chiral multiplets $Q, \widetilde{Q}$, with opposite chirality and a superpotential given by

$$
m \widetilde{Q} Q
$$

where $m$ is given by the distance of the D5- from the D7-brane in the 5 direction (together with a complex partner). As mentioned above, we also assume that when a D7-brane crosses an NS-brane, the matter and interactions are not changed. Let us indeed move the D7-brane to the left. A D5-brane is stretched between the D7 and left NS-brane. The 5 position of the D7-brane can be tuned to touch the D5-branes. Then there are three boxes of D5-branes, as in fig. 7B. 
We would like to look for fields and interactions as in eq. (3.3). Using the rules of fig. 5 and eq. (3.2), we find that in addition to the quark fields, $Q, \widetilde{Q}$, denoted by the diagonal and horizontal arrows in fig. 7B, respectively, there is another field $m$ that transforms in the bifundamental of the global symmetry, denoted by the vertical arrow in fig. 7B. The superpotential is related to the upper closed triangle of arrows and leads to eq. (3.3). We find agreement with the expectations. Let us next move the D7 to the right. By symmetry, we should find the same matter and superpotential. One difference is that the interaction now comes from a lower closed triangle. We learn that any closed triangle of arrows should contribute to the superpotential. In conclusion, by looking at the one-box case, we find agreement with the usual interaction familiar from theories with 8 supercharges.

Let us look at the two-box case. We read off the matter content from fig. 6, which consists of the expected quark fields $Q, \widetilde{Q}$ and $Q^{\prime}, \widetilde{Q}^{\prime}$ and the mass terms $m$ and $m^{\prime}$. However, in addition to these fields there is a new field $F$ that transforms in the bifundamental of the two gauge groups. The interaction can be read off from the arrows in the figure. We first note that there are two upper triangles and one lower triangle. The two upper triangles give rise to the usual interactions that are present in the one-box case. The lower one gives a new term for a configuration of D7 with more than one box. To summarize, there are three terms in the superpotential

$$
m \widetilde{Q} Q+m^{\prime} \widetilde{Q}^{\prime} Q^{\prime}+F Q^{\prime} \widetilde{Q}
$$

If we repeat the analysis for a motion of the D7-brane to the right, we get a similar term, the contribution comes from two lower triangles and one upper triangle. As a nice check of eq. (3.3) and (3.4), we can remove the middle $\mathrm{NS}^{\prime}$-brane away in the 45 directions. This gives a mass to the fields $Q^{\prime}$ and $\widetilde{Q}$ in eq. (3.4), which upon integration leads to eq. (3.3). The case with more than two boxes is generalized in an obvious way and does not give new effects.

By assuming that the D7-brane motion in the $x^{6}$ direction is an irrelevant parameter to the field theory, we conclude that the presence of a D7-brane in addition to some boxes of D5-branes gives rise to matter fields as in the previous paragraph and a superpotential given by eq. (3.4). 


\section{Interesting models and their properties}

The theories we just constructed have several non-trivial IR properties, the most interesting one being probably the fact that they can exhibit supersymmetry breaking. We hope that the techniques we just described can be useful to better understand the non-trivial dynamics of these theories. An immediate question is Seiberg's duality for these models. With the explicit realization of chiral theories using D- and NS-branes, the technique in [1,20] can be immediately applied to the models. Even more interesting would be to demonstrate that some of these models indeed break supersymmetry. We leave the real hard questions for future work, hoping that our technique will prove useful to get a better understanding of the IR properties of chiral theories, and, for the moment, we limit ourselves to some simple considerations and consistency checks.

We also want to note that up to now we assumed that all the $U(1)$ factors are frozen. Since they are generally anomalous, this must be so. However, as already noticed in [7], it may happen that, in order to get agreement between the field theory flat directions and the allowed brane motions, we have to impose their D-term equations. Analysis of specific cases may help in understanding the role of the $U(1)$ factors. However, since we expect that quantum corrections to the brane configuration have a lot to say about the fate of these $U(1)$ factors, we cannot make a general statement about them at this level. In the examples presented below, they do not seem to play any role (see however the remark at the end of section 4.2).

\subsection{Superpotential}

None of the previous models is completely defined until we say if there is a superpotential and what form this has. We will give a general rule for reading the superpotential out of the brane construction. With such a superpotential the flat directions derived from field theory are exactly matched by the allowed motions of the branes. In the cases in which the model has a known T-dual description and becomes one of those in [7], the proposal agrees with the explicit computation.

There is a simple guideline in searching for the superpotential. We do not expect any superpotential in the theories in fig. 2. The open strings giving chiral bifundamentals are localized near the intersection of the boxes. Being localized at different points, we do not expect any interaction between different bifundamentals. On the other hand, in the theories of figs. 4 and 5 , the open strings giving rise to the bifundamentals $\left(n_{i j}, \bar{n}_{i, j+1}\right)$ 
$\left(Q_{1}\right),\left(n_{i, j+1}, \bar{n}_{i+1, j+1}\right)\left(Q_{2}\right)$ and $\left(n_{i+1, j+1}, \bar{n}_{i j}\right)\left(Q_{3}\right)$ can touch at the corner of three boxes, and we can expect a superpotential. The general rule is that, every time the open strings can interact, there is indeed a superpotential. The basic building block is depicted in fig. 5 and gives rise to the superpotential

$$
W=Q_{1} Q_{2} Q_{3} .
$$

We have a superpotential for each closed cycle made up by arrows, as in fig. 5 .

The main reason for the existence of this superpotential is the fact that the flat directions predicted by the field theory analysis coincide with the allowed motion of the branes. We see many examples that demonstrate the exact matching between flat directions and motion of branes in the next sections, when we will construct interesting models.

As a consistency check, we can consider models for which the T-dual description in Type IIA as branes at orbifold singularities is known and the superpotential can be explicitly computed. In particular, the models in [7] can be realized and the results of the two methods compared. One finds complete agreement.

\subsection{The (3,2) model}

In this and the following sections, we construct several models that are supposed to break supersymmetry [21] (see [22,23, 24, 25, 26, 27, 28, 29, 30, 31, 32] for examples and discussions in the recent literature). Almost all the classical cases can be realized with the technique presented in the previous sections. One important point is that models with a particular matter content can be realized in several ways using the rules that we gave in section 3 and 4. However, different dispositions of boxes can give the same matter content but, generally, different superpotentials. Since we want models that break supersymmetry, we are interested in theories without flat directions. In the brane picture, this condition translates to the statement that no brane can move away from the system without spoiling the equilibrium or violating some charge conservation.

We also restrict our discussion to a classical analysis of the models. The study of full quantum corrections requires taking non-zero string coupling. We hope to return to this point in the future.

Consider the configuration in fig. 8. The gauge group is $S U(3) \times S U(2)$. The chiral fields are $R_{i}, i=1,2$, transforming in $(\overline{3}, 1), L$ transforms in $(1,2)$ and $Q$ in $(3,2)$. The model is anomaly-free. Using the three-box model in fig. 5, there is a superpotential

$$
W=R_{1} Q L .
$$




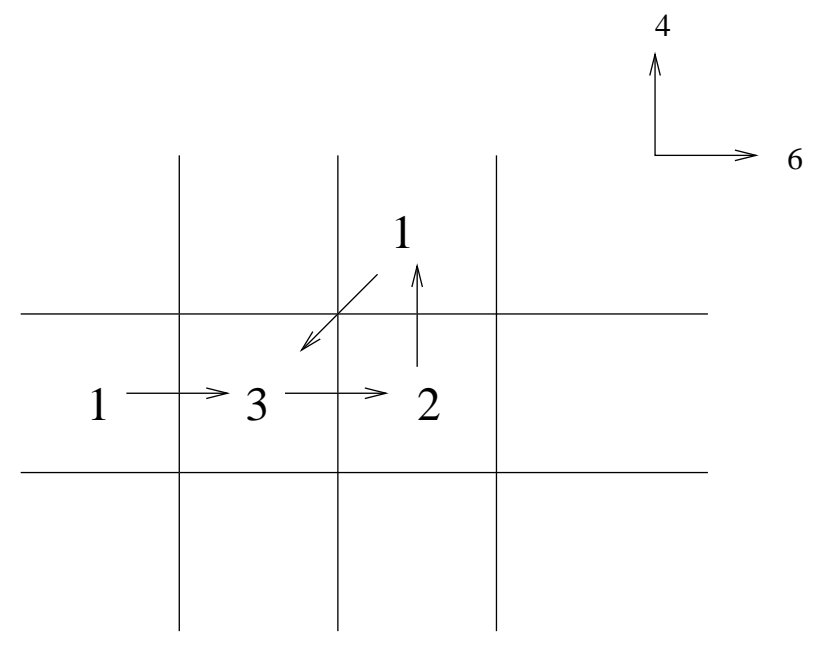

Figure 8: The $(3,2)$ model $S U(3) \times S U(2)$ with chiral fields and a superpotential.

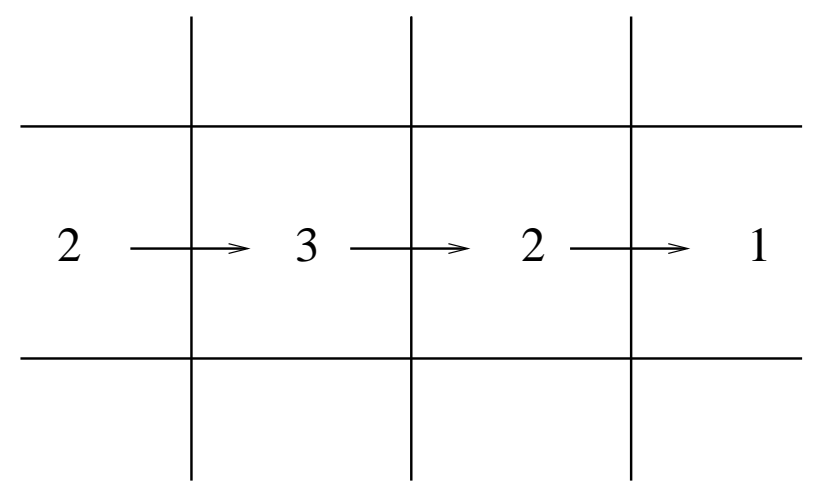

Figure 9: The $(3,2)$ model $S U(3) \times S U(2)$ without superpotential.

The model is supposed to break supersymmetry, which will be seen by taking non-zero string coupling $g_{s}$. The model has no flat directions. It is immediate to see that indeed there is no allowed motion for the branes in fig. 8 .

The particular disposition of boxes in fig. 8 is crucial. A theory with the same matter content, but without superpotential, can be realized as in fig. 9. The various fields are localized at different points and do not interact. There is no superpotential and therefore we have flat directions parametrized by the three gauge-invariant operators, $R_{1} R_{2} Q^{2}$, $R_{1} Q L$ and $R_{2} Q L$. They are reproduced by the possible brane motions: D4-branes can reconnect to an infinite one, which can move in 67 or the last two NS-branes can move with the right number of attached D4.

The model can be immediately generalized to $S U(N) \times S U(2)$ with a similar matter content (substitute 3 with $N$ in fig. 8), which is also supposed to break supersymmetry [31]. 
There is a second natural generalization of this model. Consider general integer numbers in the four boxes in fig. 8. The gauge group is $S U\left(n_{1}\right) \times S U\left(n_{2}\right)$, with a global symmetry $S U\left(m_{1}\right) \times S U\left(m_{2}\right)$. The matter content transforms in the

\begin{tabular}{|c|c|c|c|c|}
\hline & $S U\left(n_{1}\right)$ & $S U\left(n_{2}\right)$ & $S U\left(m_{1}\right)$ & $S U\left(m_{2}\right)$ \\
\hline$R$ & $\square$ & 1 & 1 & $\square$ \\
\hline$Q$ & $\square$ & $\square$ & 1 & 1 \\
\hline$L$ & 1 & $\square$ & 1 & $\square$ \\
\hline$R^{\prime}$ & $\square$ & 1 & $\square$ & 1 \\
\hline
\end{tabular}

Anomaly cancellation requires $m_{1}+m_{2}=n_{2}$ and $n_{1}=m_{2}$. The superpotential is given by

$$
W=R Q L .
$$

There is a range of values for $n_{1}$ and $n_{2}$ for which the brane construction lifts all the flat directions. From the field theory point of view, baryonic flat directions may survive the introduction of the superpotential (4.3) (see [28,29]). Here the $U(1)$ factors that we assumed to be frozen may play a role. It would be interesting to understand this point better and to study if these theories break supersymmetry as the $(3,2)$ model does.

In general, it is quite easy to construct brane configurations in which there is no allowed motion for the branes and, therefore, there are no expected flat directions. All these theories are, in principle, good candidates for models that break supersymmetry.

\subsection{Three-box models}

Just to provide more examples, we can classify all the models that can be obtained by arranging configurations of three boxes. Figure 10 presents all possible cases. Cases $b, d, f$ are excluded, as they are anomalous. Cases $a, c, e$ have a gauge group $S U(n)$ with $n_{f}$ flavours. Case $c$ has no superpotential. Cases $c$ and $e$ have a superpotential that gives mass to the quark fields.

To this classification, we can add an orientifold plane $\mathrm{O} 7$ or $\mathrm{O}^{\prime}$ and produce more models. The orientifold planes can be put either on top of an NS-brane or not. This procedure can be repeated for a configuration of four boxes and so on. We expect that a large class of models can be analysed systematically, using this classification in terms of branes. 
a

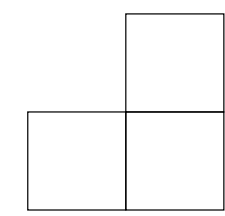

b

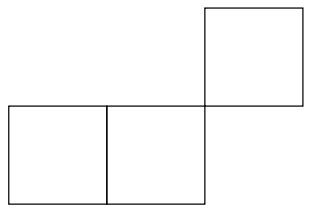

c

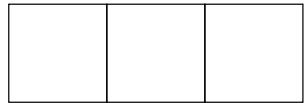

$\mathrm{d}$
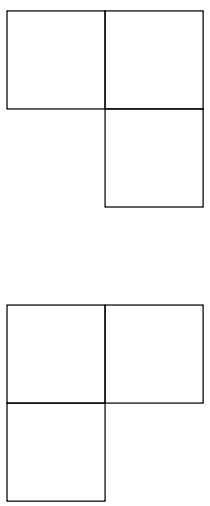

$\mathrm{f}$

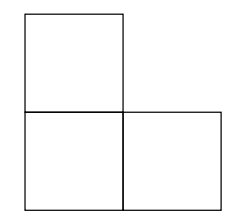

Figure 10: Various cases of three-box models.

\section{4. $S U(N)$ with antisymmetric}

We are interested in an $S U(N)$ gauge theory with an antisymmetric tensor representation $A, F$ fundamentals and $N+F-4$ antifundamentals. There are several ways for realizing this theory, according to whether or not we want a superpotential. The crucial ingredient is an NS-brane stuck at an orientifold plane, according to the rules in section 2.

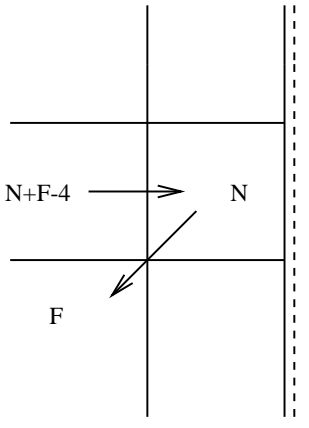

a)

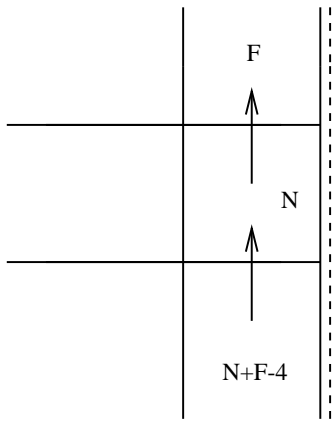

b)

Figure 11: Different realizations for $S U(N)$ with an antisymmetric, $F$ fundamentals and $N+F-4$ antifundamentals.

Consider two possible realizations. The case a) in fig. 11 has no superpotential, while the case in figure b) has a superpotential of the form

$$
W=A \bar{Q} \bar{Q} .
$$


This is because the antisymmetric tensor arises from open strings localized at the orientifold plane and they can interact with the antifundamental representations only in case b) of fig. 11.

Let us analyse the interesting case $F=0$. In case a), we have flat directions, which correspond to reconnecting D5-branes into infinite ones and moving them in 67 . Due to the presence of the orientifold plane, we can only move away an even number of D5-branes. The theory can be higgsed to $U S p(4)$ for $N$ even and $S U(5)$ for $N$ odd. This agrees with the field theory expectations [21]. For $N$ odd, the model, when equipped with the superpotential (4.4), is supposed to break supersymmetry. We can check that in case b) of fig. 11 (for $F=0$ ) there is only one allowed motion of branes: we can move away the NS-brane stuck at the orientifold plane. However, the resulting configuration (see fig. 3) requires an even number of D5-branes to live at the orientifold. This means that there exist flat directions only when $N$ is even. This is in agreement with the complete lifting of the flat directions in field theory and the conjectured susy breaking when $N$ is odd.

\subsection{Chiral non-chiral theories}

In this section we show that, even in our classical approximation with zero string coupling, we can say something about the non-trivial IR properties of some models.

It is known 33 that, in order to study the IR properties of a model, a chiral theory can be expanded into a non-chiral theory with more gauge factors but, sometimes, with simpler properties. For example, $S U(n)$ with an antisymmetric and $n_{f}=n-4$ antifundamentals can be equivalently described using the non-chiral theory $U S p(n-4) \times S U(n)$ 国 with a chiral bifundamental and $n-4$ antifundamentals for $S U(n)$. At strong coupling the $U S p$ group confines, and the mesons of the theory, which completely saturate the anomaly, reproduce the antisymmetric for $S U(n)$. The non-chiral model can be easily realized in terms of branes. It indeed belongs to the infinite series of models in (2.8).

Consider in fact, as in fig. 12, the case with two NS-branes not at the orientifold point. Stretch $n-4$ D5-branes between the first NS and its image. Using rule (b), we need $n$ D5-branes between the first and the second NS. Using rule (a), we need to put also $n-4$ semi-infinite D5-branes. The equivalence between the chiral and the non-chiral

4 This theory can be considered chiral in the sense that the bifundamental and the $n-4$ fundamentals are chiral, but the number of fundamentals and antifundamentals for $S U(n)$ is the same. 

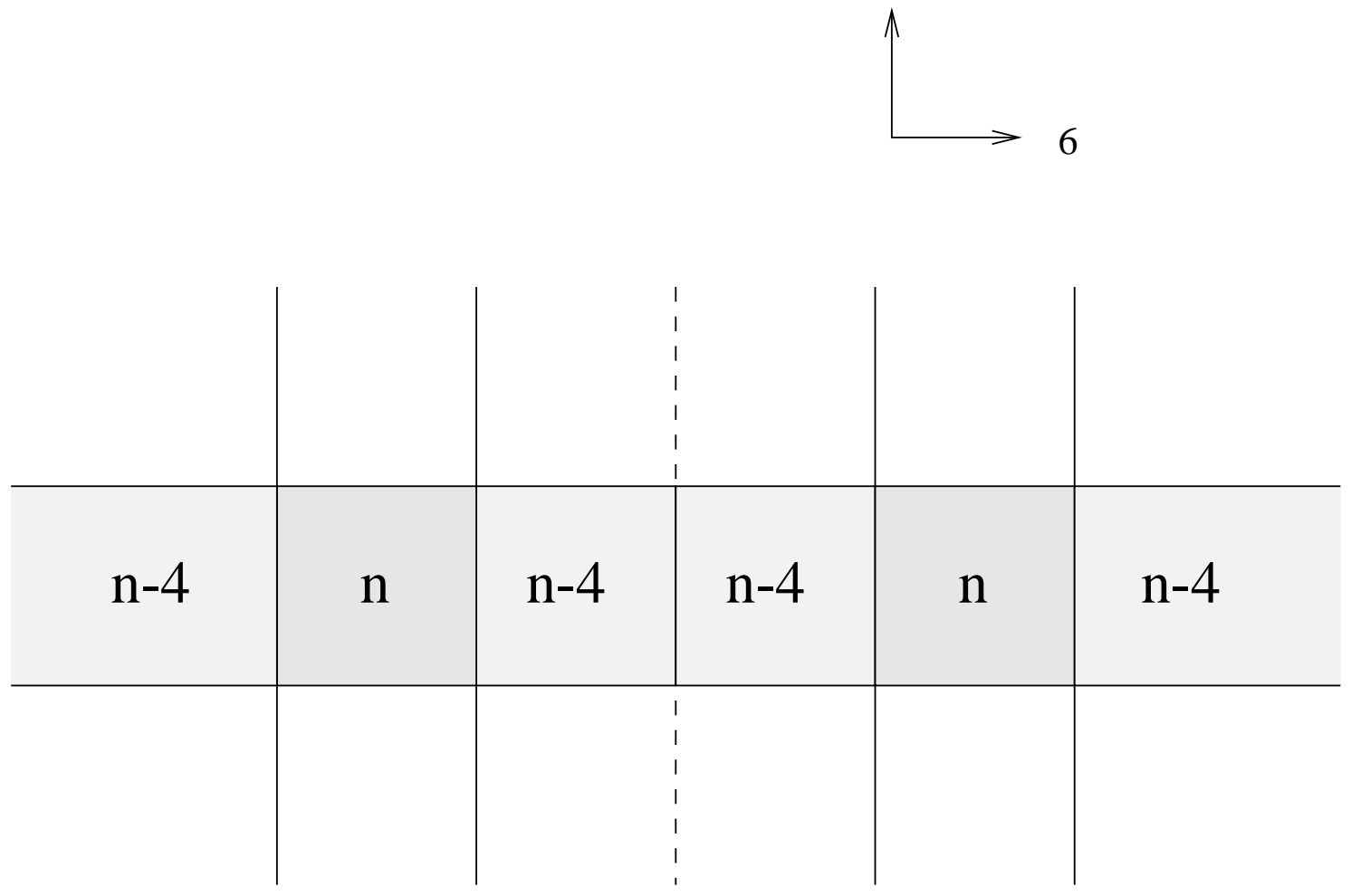

Figure 12: Brane realization of $U S p(n-4) \times S U(n)$ gauge group with a bifundamental and a superpotential $W=0$.

model can be easily shown by moving the first NS-brane toward the orientifold. When the NS-brane together with its image touch the orientifold, the USp coupling constant flows to infinite value. One of the two NS-branes at the orientifold is now free to move in the 789 directions. We move it to large 789 positions. The resulting theory is therefore $S U(n)$ with an antisymmetric and $n_{f}=n-4$ antifundamentals.

A similar mechanism was discussed in [13] in the context of $(0,1)$ six-dimensional theories to explain the small instanton transition in which a tensor multiplet is traded with 29 hypermultiplets. This phenomenon was first discovered in the case of the small $E_{8} \times E_{8}$ instanton, which has an interpretation as a M-theory five-brane which has left the boundary, when the $E_{8} \times E_{8}$ heterotic string is interpreted as M-theory on $S^{1} / Z_{2}$. Also the theory of $S O(32)$ small instantons at spacetime singularities sometimes has a Coulomb branch parametrized by tensor multiplets [11]. If we discard the two $\mathrm{NS}^{\prime}$-branes in the models considered before and perform T-duality in two directions we exactly recover the theory of $S O(32)$ small instantons at spacetime singularities as described in [13]. As shown there, even in this case, the small instanton transition can be interpreted as a fivebrane that has left the boundary (in this case an $\mathrm{O} 8$ orientifold plane). The mechanism 
is essentially the same as the one described above to demonstrate the equivalence between chiral and non-chiral models

It was shown in 34 that the small instanton transition, when the theory is further compactified to a four-dimensional $N=1$ model, can give rise to a transition in which the net number of generations changes. We have explicitly seen that a non-chiral model can be connected to a chiral one with a mechanism related by T-duality to the six-dimensional small instanton transition. The results in [34 and those described above seem to suggest that the relation between the small instanton transition and the physics of chirality-changing transition in four dimensions is quite general and follows from the same universal effect. It would be interesting to check the relation between the two approaches and to learn more about such transitions.

Other IR results, which we expect to be able to obtain within the classical approximation, concern Seiberg's duality. We expect that the techniques in [20] can be applied to our brane construction.

\section{Conclusions}

In this paper we have presented a general setup for constructing four-dimensional gauge theories, which are generically chiral. From the examples that we presented in the previous sections, the rules for constructing models should now be clear. Using these rules, we can construct quite a lot of the models present in the literature. Many of them are supposed to break supersymmetry. Other models, not treated in the literature, can be studied systematically. It is also easy to construct general models without flat directions, which may break supersymmetry. Moreover, the brane construction arranges the various models into families that can be treated in a unified way. Different dispositions of boxes gives rise to different superpotentials. In this paper we only discussed the superpotential, which is naturally present in the brane configuration. However, more general interactions can be obtained by introducing more ingredients in the picture, for example rotations for some branes.

We hope that the flexibility of this construction can be useful for constructing and studying models when the field theory analysis gets complicated. In this paper we limited ourselves to a classical analysis, but, even at the classical level, the brane construction

\footnotetext{
5 Notice that the argument does not use in any way the existence of the NS'-branes.
} 
can give a help in finding the moduli space of flat directions. The issue of supersymmetry breaking can be addressed only when we turn on the coupling constant, which introduces crucial differences and bending in the branes configuration. New tools are needed to study the quantum theory. We hope to return to this subject, clearly the most interesting one, in the future.

\section{Acknowledgements}

We would like to thank O. Aharony, K. S. Babu, J. H. Brodie, B. Kol, L. Randall, R. Rattazzi and M. Strassler for useful discussions. 


\section{References}

[1] A. Hanany and E. Witten, Type IIB Superstrings, BPS Monopoles, And ThreeDimensional Gauge Dynamics, IASSNS-HEP-96/121, hep-th/9611230.

[2] J.H. Brodie and A. Hanany, Type IIA Superstrings, Chiral Symmetry, and $N=14 D$ Gauge Theory Duality, hep-th/9704043

[3] A. Hanany and A. Zaffaroni, Chiral Symmetry from Type IIA Branes, hep-th/9706047.

[4] K. Landsteiner, E. Lopez and D. A. Lowe, Duality of Chiral N=1 Supersymmetric Gauge Theories via Branes, hep-th/9801002.

[5] I. Brunner, A. Hanany, A. Karch and D. Lüst, Brane Dynamics and Chiral non-Chiral Transitions, hep-th/9801017.

[6] S. Elitzur, A. Giveon, D. Kutasov and D. Tsabar, Branes, Orientifolds and Chiral Gauge Theories, hep-th/9801020.

[7] J. Lykken, E. Poppitz and S. P. Trivedi, Chiral Gauge Theories from D-Branes, hepth/9708134.

[8] J. Lykken, E. Poppitz and S.P. Trivedi, M(ore) on Chiral Gauge Theories from DBranes, hep-th/9712193.

[9] M. R. Douglas and G. Moore, D-branes, Quivers, and ALE Instantons, hepth/9603167.

[10] N. Evans, C. V. Johnson and A. D. Shapere, Orientifolds, Branes, and Duality of $4 D$ Gauge Theories, hep-th/9703210.

[11] K. Intriligator, New String theories in Six Dimensions via Branes at Orbifold Singularities, hep-th/9708117.

[12] J. D. Blum and K. Intriligator, New Phases of String Theory and 6d RG Fixed Points via Branes at Orbifold Singularities, hep-th/9705044.

[13] A. Hanany and A. Zaffaroni, Branes and Six-Dimensional Supersymmetric Theories, hep-th/9712145.

[14] I. Brunner and A. Karch, Branes at Orbifolds versus Hanany Witten in Six Dimensions, hep-th/9712143.

[15] J. de Boer, K. Hori, H. Ooguri and Y. Oz, Branes and Dynamical Supersymmetry Breaking, hep-th/9801060.

[16] O. Aharony and A. Hanany, Branes, Superpotentials and Superconformal Fixed Points, hep-th/9704170.

[17] O. Aharony and A. Hanany, unpublished.

[18] O. Aharony, A. Hanany and B. Kol, Webs of $(p, q)$ 5-branes, Five Dimensional Field Theories and Grid Diagrams, hep-th/9710116.

[19] K. Landsteiner and E. Lopez, New Curves from Branes, hep-th/9708118.

[20] S. Elitzur, A. Giveon and D. Kutasov, Branes and $N=1$ Duality in String Theory, hep-th/9702014. 
[21] I. Affleck, M. Dine and N. Seiberg, Dynamical Supersymmetry Breaking In Four Dimensions And Its Phenomenological Implications, Nucl. Phys. B256 (1985) 557.

[22] H. Murayama, Studying Non-calculable Models of Dynamical Supersymmetry Breaking Phys. Lett. B355 (1995) 187, hep-th/9505082.

[23] M. Dine, A. E. Nelson, Y. Nir and Y. Shirman, New Tools for Low Energy Dynamical Supersymmetry Breaking Phys. Rev. D53 (1996) 2658, hep-ph/9507378.

[24] E. Poppitz and S. Trivedi, Some Examples of Chiral Moduli Spaces and Dynamical Supersymmetry Breaking, Phys. Lett. B365 (1996) 125, hep-th/9507169.

[25] P. Pouliot, Duality in SUSY SU $(N)$ with an Antisymmetric Tensor, Phys. Lett. B367 (1996) 151, hep-th/9510148.

[26] K. Intriligator and S. Thomas Dynamical Supersymmetry Breaking on Quantum Moduli Spaces Nucl. Phys. B473 (1996) 121, hep-th/9603158.

[27] C. Csaki, L. Randall and W. Skiba, More Dynamical Supersymmetry Breaking,Nucl. Phys. B479 (1996) 65, hep-th/9605108.

[28] E. Poppitz, Y. Shadmi and S. P. Trivedi Duality and Exact Results in Product Group Theories, Nucl. Phys. B480 (1996) 125, hep-th/9605113.

[29] E. Poppitz, Y. Shadmi and S. P. Trivedi Supersymmetry Breaking and Duality in $S U(N) x S U(N-M)$ Theories, Phys. Lett. B388 (1996) 561, hep-th/9606184.

[30] C. Csaki, L. Randall, W. Skiba and R. Leigh, Supersymmetry Breaking Through Confining and Dual Theory Gauge Dynamics, Phys. Lett. B387 (1996) 791, hepth/9607021.

[31] K. Intriligator and S. Thomas Dual Descriptions of Supersymmetry Breaking, hepth/9608046.

[32] R. G. Leigh, L. Randall and R. Rattazzi Unity of Supersymmetry Breaking Models, Nucl. Phys. B501 (1997) 375, hep-ph/9704246.

[33] M. Berkooz, The Dual of Supersymmetric SU(2k) with an Antisymmetric Tensor and Composite Dualities, hep-th/9505067, Nucl. Phys. B452 (1995) 513.

[34] S. Kachru and E. Silverstein, Chirality Changing Phase Transitions in 4d String Vacua, Nucl. Phys. B504 (1997) 272, hep-th/9704185. 